\title{
Dynamic chaotic gravitational search algorithm-based kinetic parameter estimation of hepatocellular carcinoma on ${ }^{18} \mathrm{~F}-\mathrm{FDG} \mathrm{PET} / \mathrm{CT}$
}

Jianfeng $\mathrm{He}^{1}$, Tao Wang ${ }^{1}$, Yongjin Li $i^{1}$, Yinglei Deng ${ }^{2}$ and Shaobo Wang ${ }^{2^{*}}$

\begin{abstract}
Background: Kinetic parameters estimated with dynamic ${ }^{18} \mathrm{~F}$-FDG PET/CT can help to characterize hepatocellular carcinoma (HCC). We aim to evaluate the feasibility of the gravitational search algorithm (GSA) for kinetic parameter estimation and to propose a dynamic chaotic gravitational search algorithm (DCGSA) to enhance parameter estimation.
\end{abstract}

Methods: Five-minute dynamic PET/CT data of $20 \mathrm{HCC}$ s were prospectively enrolled, and the kinetic parameters $k_{1} \sim k_{4}$ and the hepatic arterial perfusion index (HPI) were estimated with a dual-input three-compartment model based on nonlinear least squares (NLLS), GSA and DCGSA.

Results: The results showed that there were significant differences between the HCCs and background liver tissues for $k_{1}, k_{4}$ and the HPI of NLLS; $k_{1}, k_{3}, k_{4}$ and the HPI of GSA; and $k_{1}, k_{2}, k_{3}, k_{4}$ and the HPI of DCGSA. DCGSA had a higher diagnostic performance for $k_{3}$ than NLLS and GSA.

Conclusions: GSA enables accurate estimation of the kinetic parameters of dynamic PET/CT in the diagnosis of HCC, and DCGSA can enhance the diagnostic performance.

Keywords: Kinetic models, PET/CT, Hepatocellular carcinoma, Gravitational search algorithm

\section{Background}

Hepatocellular carcinoma ( $\mathrm{HCC})$ is the third leading cause of cancer-related death, with insignificant clinical manifestations and concealed symptoms at the initial stage of the disease $[1,2]$. Conventional medical imaging techniques such as computed tomography (CT) and magnetic resonance imaging (MRI) are often used for the initial examination in clinical practice. However, they can only generate structural images and lack tumor metabolic information [3]. Positron emission tomography (PET)/ $\mathrm{CT}$ has emerged as a noninvasive functional imaging

\footnotetext{
*Correspondence: wshbo_98@126.com

2 PET/CT Center, Affiliated Hospital of Kunming University of Science and Technology, First People's Hospital of Yunnan, Kunming 650031, China

Full list of author information is available at the end of the article
}

method that allows assessment of metabolic function in tumors by injecting glucose analogs as radiotracers [4].

Although static ${ }^{18}$ F-FDG PET imaging obtains several parameters, such as standard uptake values (SUV), metabolic tumor volume and total lesion glycolysis, it is insufficient to describe the metabolic processes of ${ }^{18} \mathrm{~F}$-FDG [5]. Dynamic PET/CT imaging can track the distribution of ${ }^{18} \mathrm{~F}$-FDG in tissues and derive some kinetic parameters that accurately describe the cellular metabolic processes of ${ }^{18} \mathrm{~F}$-FDG to enhance diagnosis and therapy in various diseases, and compartmental modeling is routinely applied to estimate kinetic parameters [6, 7]. Sarkar et al. [8] demonstrated that dynamic ${ }^{18}$ F-FDG PET with tracer kinetic modeling has the potential to diagnose nonalcoholic steatohepatitis. Wang et al. [9] found that dynamic ${ }^{18}$ F-FDG PET with optimization-derived blood input 
function kinetic modeling can effectively distinguish liver lesions. Considering 60-min or more dynamic PET/CT is not easily available in routine clinical settings. Samimi et al. [10] reported that 5-min dynamic PET / CT plus a static PET/CT enables accurate and robust estimation of kinetic parameters in patients with liver metastases. Our previous studies $[11,12]$ also demonstrated that 5 -min dynamic ${ }^{18}$ F-FDG PET/CT can provide blood flow and metabolic information that enhances the detection of HCC lesions [11], and derived perfusion and earlyuptake PET/CT are feasible for diagnosing HCC and provide added functional parameters to enhance diagnostic performance [12].

Nonlinear least squares (NLLS) is commonly used to estimate compartment model parameters [10, 12-14], and its essence is to minimize the sum of squared errors of the measured and estimated values; however, NLLS easily falls into the local optimum in the process of parameter estimation, and the set initial value has a great influence on the results [15]. The gravitational search algorithm (GSA) is a swarm intelligent optimization algorithm that is based on Newtonian gravity and has a strong searching ability [16]. It is more stochastic, does not require initial values, and can perform better parameter estimation compared to NLLS; however, whether GSA can function well to estimate compartment model parameters with dynamic ${ }^{18} \mathrm{~F}$-FDG PET/CT is unclear. Additionally, a dynamic chaotic gravitational search algorithm (DCGSA) is proposed to improve the exploration ability and global search ability to enhance parameter estimation. Therefore, this study evaluated the role of the compartmental parameters of 5 -min ${ }^{18} \mathrm{~F}$-FDG PET/CT estimated by NLLS, GSA and DCGSA for distinguishing $\mathrm{HCC}$ from background liver tissue.

\section{Methods}

\section{Patients}

This study was approved by the Institutional Review Committee (IRB) of the First People's Hospital of Yunnan Province (No. 2017YYLH035), informed consent was obtained from all the patients, and all the methods were performed in accordance with the Declaration of Helsinki.

We recruited 28 patients with clinically suspected HCC, and a 5-min dynamic PET/CT scan was added before conventional PET/CT. Ten patients were excluded because they had a non-HCC pathological diagnosis $(\mathrm{n}=5)$, lacked a pathological diagnosis $(\mathrm{n}=3)$, and had suboptimal imaging quality $(\mathrm{n}=2)$.

Eighteen patients (17 males and 1 female) who had pathologically confirmed HCC were finally included in this study. Sixteen patients had a single lesion, and two patients had two lesions. A total of $20 \mathrm{HCC}$ tumors that were confirmed by surgery $(n=14)$ or biopsy $(n=6)$ were used in this study, and the long axis of these tumors was $1.9-15.0 \mathrm{~cm}$ (average $6.5 \pm 3.6$ ).

\section{Dynamic PET/CT}

All examinations were performed using a Philips Ingenuity TF PET/CT scanner (Cleveland, OH, USA), and a Philips IntelliSpace Portal v7.0.4.20175 was used for postprocessing. In summary, after the patients had fasted for at least $6 \mathrm{~h}$, blood glucose was verified. A low-dose liver CT scan $(120 \mathrm{kV}, 100 \mathrm{mAs})$ was performed for attenuation correction and image fusion. A 5-min dynamic PET scan was performed over the liver region after intravenous administration of $5.5 \mathrm{MBq} / \mathrm{kg}{ }^{18} \mathrm{~F}$-FDG. Dynamic PET data were divided into 16 frames using the following sampling schedule: 12 frames of $5 \mathrm{~s}$ and 4 frames of $60 \mathrm{~s}$ each. Dynamic PET images were then reconstructed using the standard ordered subsets expectation maximization (OSEM) algorithm.

Regions of interest (ROIs) were drawn manually in the CT images of each patient, including the HCC, background liver tissue, aorta and portal vein (the extrahepatic portal vein rather than the intrahepatic portal vein), with manual slice-by-slice adjustment. An ROI was copied to the PET/CT images after image fusion, and time activity curves (TACs) consisting of the maximum SUV (SUVmax) extracted from each frame were generated. Figure 1 shows the ROIs drawn on a transaxial dynamic $\mathrm{PET} / \mathrm{CT}$ image of a patient with HCC.

\section{Kinetic modeling}

A dual-input three-compartment model was used to assess the steady-state hepatic metabolism of ${ }^{18} \mathrm{~F}$-FDG, as shown in Fig. 2 [17, 18].

In Fig. 2, $k_{1}(\mathrm{ml} / \mathrm{min} / \mathrm{ml})$ represents the rate constant of ${ }^{18} \mathrm{~F}$-FDG from the blood to the liver tissue, and $k_{2}$ represents the clearance rate back to the blood. $k_{3}$ is the rate constant of further phosphorylation of ${ }^{18} \mathrm{~F}$-FDG to ${ }^{18} \mathrm{~F}$-FDG-6-phosphate and $k_{4}$ is the dephosphorylation rate of phosphatase. $C_{B}(t)$ represents the ${ }^{18}$ F-FDG concentration in the blood:

$$
C_{B}(t)=H P I \times A(t)+(1-H P I) \times P(\mathrm{t}),
$$

where $A(t)$ represents the ${ }^{18} \mathrm{~F}$-FDG concentration in the hepatic artery, and $P(t)$ represents the ${ }^{18}$ F-FDG concentration in the portal vein. The HPI represents the hepatic artery perfusion index (the ratio of arterial blood volume to total blood volume). $C_{M}(t)$ represents the free-state ${ }^{18} \mathrm{~F}$-FDG concentration and the metabolized ${ }^{18} \mathrm{~F}$-FDG-6-phosphate concentration in the liver tissue compartment. $C_{T}(t)$ represents the curve of the tracer concentration in the tissue measured from the PET image over time and is the output function of the kinetic model: 

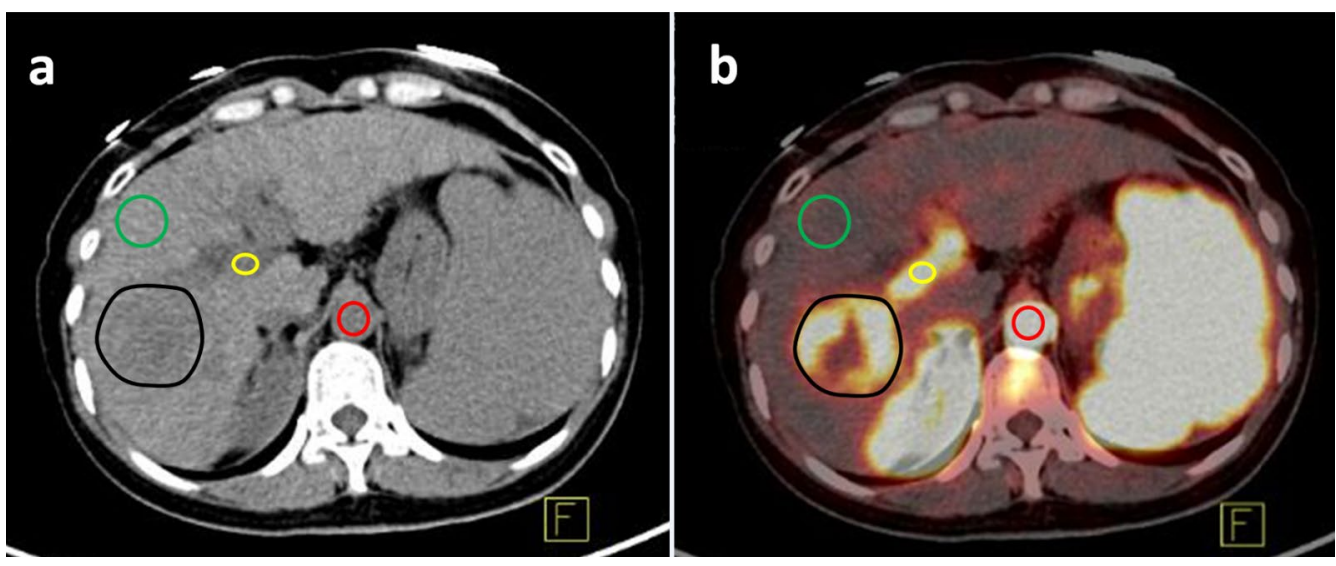

Fig. 1 Region of interest drawn in dynamic PET/CT. a CT image, b PET/CT fusion image. The HCC is shown in the black circle, background liver tissue is shown in the green circle, the portal vein is shown in the red circle, and the aorta is shown in the yellow circle. Blood ${ }^{18} \mathrm{~F}-\mathrm{FDG}$ enters the aorta, portal vein, spleen and HCC
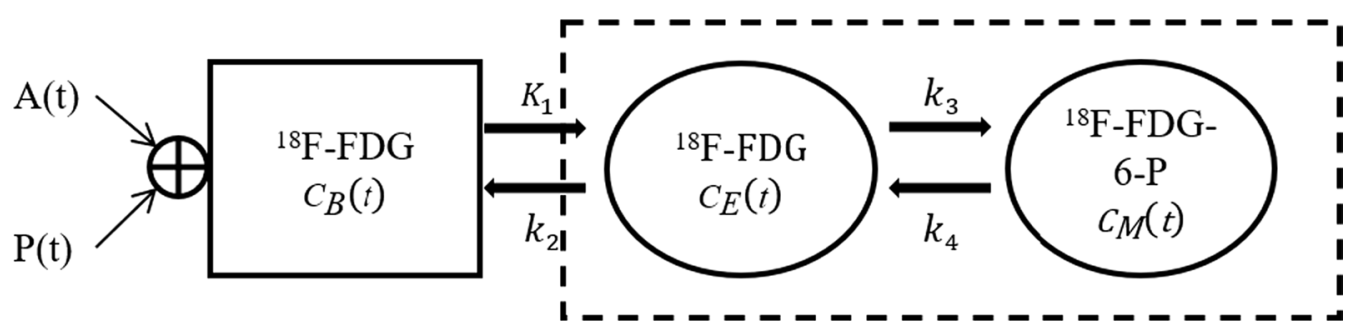

Fig. 2 A dual-input three-compartment model

$$
C_{T}(\mathrm{t})=\frac{K_{1}}{\alpha_{2}-\alpha_{1}} \times\left[\left(k_{3}+k_{4}-\alpha_{1}\right) e^{-\alpha_{1} t}+\left(\alpha_{2}-k_{3}-k_{4}\right) e^{-\alpha_{2} t}\right] \otimes C_{B}(t)
$$

where $\alpha_{2}$ and $\alpha_{1}$ can be described as follows:

$$
\begin{aligned}
& \alpha_{1}=\frac{k_{2}+k_{3}+k_{4}-\sqrt{\left(k_{2}+k_{3}+k_{4}\right)^{2}-4 k_{2} \times k_{4}}}{2} \\
& \alpha_{2}=\frac{k_{2}+k_{3}+k_{4}+\sqrt{\left(k_{2}+k_{3}+k_{4}\right)^{2}-4 k_{2} \times k_{4}}}{2}
\end{aligned}
$$

\section{Estimation of the kinetic parameters}

This study proposes an improved algorithm, the DCGSA, for the estimation of liver kinetic parameters. In the GSA, a solution space about the objective function is initialized randomly, with each individual in the space as one feasible solution in the objective function. With constant movement, individuals will move toward the most mass, which is the optimal solution of the search space [16].
The convergence speed of the GSA is faster, which makes it fall into a local optimum without global search [19].

In the DCGSA, the dynamic adjustment strategy is introduced for the gravitational constant of GSA to improve the algorithm exploration capacity and mining capacity. At the same time, inertia weights and chaotic sequences are added to the particle speed update process to avoid falling into a local optimum and to improve the global search ability. The overall flow of the DCGSA is shown in Fig. 3. The DCGSA can be simply divided into four parts: the initialization phase, the evaluation phase, the acceleration phase, and the update position phase.

\section{Initialization phase}

To start the search process for the DCGSA, the initial population of $N$ individuals is randomly generated in the search space, which represents a set of model parameters $\left(k_{1}, k_{2}, k_{3}, k_{4}\right.$ and the HPI). The position of each particle is represented by the following: 


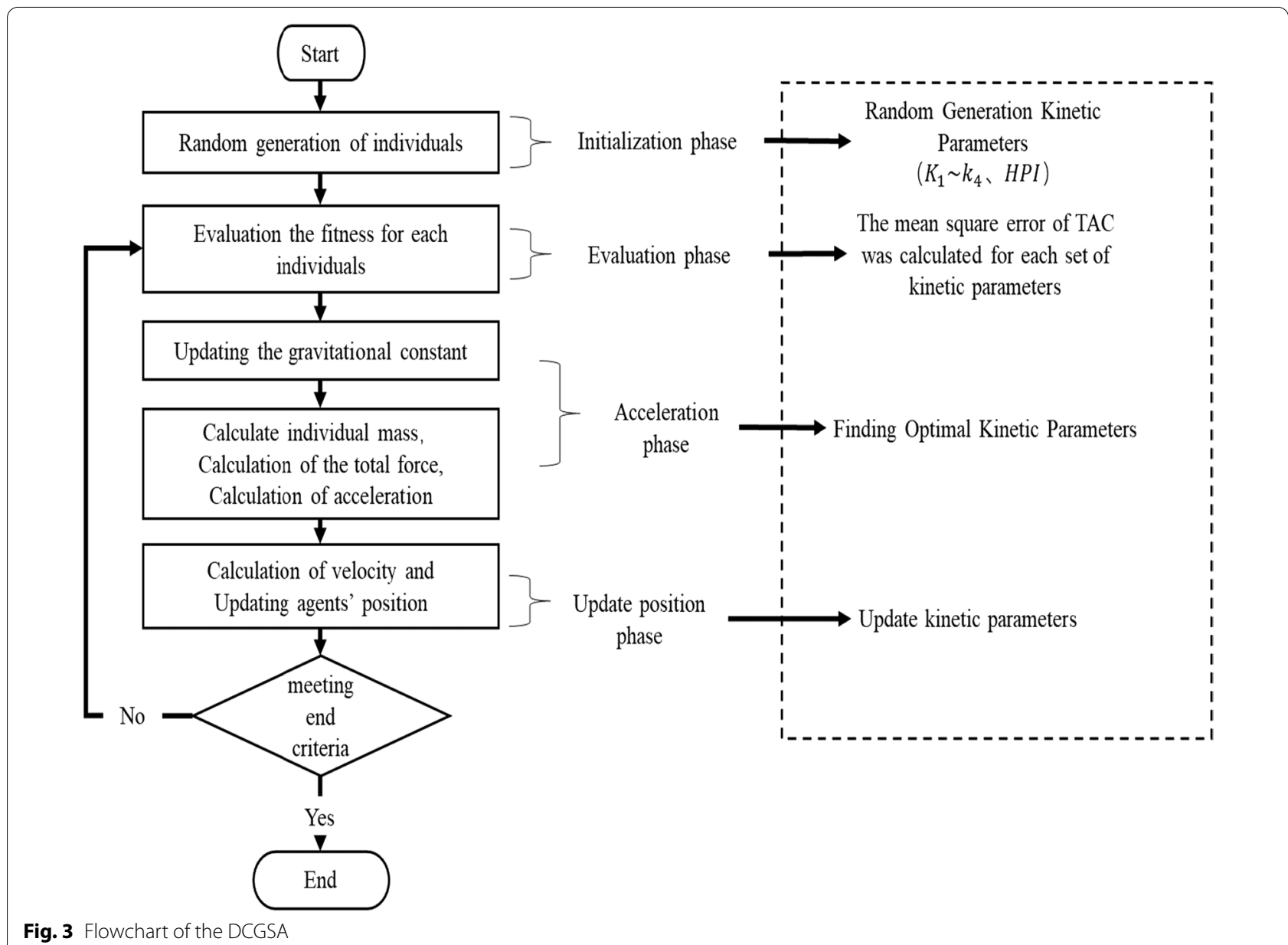

Fig. 3 Flowchart of the DCGSA

$$
X_{i}=\left(x_{i}^{1}, x_{i}^{2}, \cdots, x_{i}^{d}, \cdots, x_{i}^{D}\right) \quad \text { for } i=1, \cdots, N
$$

where $x_{i}^{D}$ is the position of the $i$ th individual in the $\mathrm{D}$ dimension.

\section{Evaluation phase}

The individual is evaluated according to the fitness function, defined as the sum of the squared errors squared errors of the experimental data and the fitted data:

$$
f i t_{i}(t)=\sum_{i=1}^{N}\left(C_{T}{ }^{\prime}(t)-C_{i}^{m}(t)\right)^{2},
$$

where $N$ is the number of individuals, $i$ is the index, $C(t)$ is the actual concentration of ${ }^{18} \mathrm{~F}-\mathrm{FDG}$ in the obtained tissue, and $C_{i}^{m}(t)$ is the estimated concentration of ${ }^{18} \mathrm{~F}$ FDG in the obtained tissue. After that, the individual continuously updates its inertial mass $M_{i}(t)$ during movement by the following equations, that is, to find the best model parameters in the search space:

$$
\begin{aligned}
& m_{i}(t)=\frac{f i t_{i}(t)-\operatorname{worst}(t)}{\operatorname{best}(t)-\operatorname{worst}(t)} \\
& M_{i}(t)=\frac{m_{i}(t)}{\sum_{j=1}^{N} m_{j}(t)}
\end{aligned}
$$

where $\operatorname{best}(t)$ and worst $(t)$ are the best and worst fitness, respectively:

$$
\begin{aligned}
& \operatorname{best}(t)=\min _{\mathrm{j} \in 1, \cdots, N} \mathrm{fit}_{\mathrm{j}}(\mathrm{t}) \\
& \operatorname{worst}(t)=\max _{\mathrm{j} \in\{1, \cdots, N\}} \mathrm{fit}_{\mathrm{j}}(\mathrm{t})
\end{aligned}
$$




\section{Acceleration phase}

The individual will be subjected to the force of other individuals in the search space. Based on the law of universal gravitation, the force $\mathrm{F}_{\mathrm{ij}}^{\mathrm{d}}(\mathrm{t})$ of individual $i$ by individual $j$ is:

$$
F_{i j}^{d}(t)=G(t) \frac{M_{i}(t) \times M_{j}(t)}{R_{i j}(t)+\varepsilon}\left(x_{i}^{d}(t)-x_{j}^{d}(t)\right)
$$

where $M_{i}(t)$ and $M_{j}(t)$ are the inertial masses of individuals $i$ and $j$ at time $t$, respectively, that is, the TACs calculated by a set of kinetic parameters. $\epsilon$ is a small value to prevent errors. $R_{i j}(t)$ is the Euclidean distance between individuals $i$ and $j . G(t)$ is a gravitational constant that decreases with time $t$ and is described by Eq. (12); it can affect the force and acceleration of the individual:

$$
G(t)=G_{0} e^{-\alpha \frac{t}{T}}
$$

where $\mathrm{G}_{0}$ is an initial value, $\alpha$ is a constant, $t$ is the current number of iterations, and $T$ is the maximum number of iterations. The traditional gravitational constant was not fully explored in the early stage of iteration, and fell into a local optimum [20]. Lei et al. [21] introduced a self-adaptive gravitational constant, and Seyedali et al. [22] used chaotic mapping to adjust the gravitational constant.

Therefore, this paper proposes a new gravitational constant expression, introducing an improved dynamic adjustment strategy, which contains a random variable as follows:

$$
G^{\prime}(t)=G_{0} e^{-\alpha \frac{t}{T^{1.5}} \times\left(\operatorname{rand}_{t}+\frac{t}{T}\right)},
$$

where rand $\mathrm{t}_{\mathrm{t}}$ is a random number in $(0,1) . G^{\prime}(t)$ adopts a large step and long movement in the early stage of iteration to increase the particle exploration ability and enough time for optimization. Random variables can abruptly change the gravitational constant during iterations, improving the ability of particles to jump out of the local optimum. The process of moving in small steps is adopted in the later stage of iteration, which effectively avoids the premature convergence of particles and improves the mining capacity.

The resultant force of each particle is calculated as follows:

$$
F_{i}^{d}(t)=\sum_{j \in K \text { best }, j \neq i} \operatorname{rand}_{j} F_{i j}^{d}(t)
$$

where Kbest decreases with the number of iterations, the initial value is $N$, and rand $d_{j}$ is a random number between the interval $(0,1)$. The acceleration of particle $i$ at time $t$ is calculated as follows:

$$
a_{i}^{d}(t)=\frac{F_{i}^{d}(t)}{M_{i}(t)}
$$

\section{Update position phase}

In each iteration, the particles update their velocity and position as follows:

$$
\begin{aligned}
& v_{i}^{d}(t+1)=\operatorname{rand}_{i} \times v_{i}^{d}(t)+a_{i}^{d}(t) \\
& x_{i}^{d}(t+1)=x_{i}^{d}(t)+v_{i}^{d}(t+1)
\end{aligned}
$$

where $\operatorname{rand}_{\mathrm{i}}$ is a uniformly distributed random number between the interval $(0,1)$. However, some particles move too fast in the moving process, thus flying out of the solution space. Li et al. [23] introduced inertia weight instead of random variables to restrict the particle velocity. Gao et al. [24] replaced random variables with chaotic sequences. Therefore, this paper adds inertia weight and chaotic sequence to the particle speed updating process to further limit the particle speed:

$$
\begin{aligned}
& v_{i}^{d}(t+1)=W^{d}(t) \times v_{i}^{d}(t)+a_{i}^{d}(t) \\
& x_{i}^{d}(t+1)=x_{i}^{d}(t)+v_{i}^{d}(t+1) \times c(i) \\
& W^{d}(t)=\omega_{\max }-\frac{\omega_{\max }-\omega_{\min }}{T} \times t,
\end{aligned}
$$

where $\omega_{\max }$ and $\omega_{\min }$ are the inertia weights $\left(\omega_{\max }=0.7\right.$, $\omega_{\min }=0.1$, which is mainly based on experience), and $c(i)$ is a chaotic sequence. A larger inertia weight can improve the exploration ability, and a smaller inertia weight can improve the mining ability. Moreover, the ergodicity and dynamics of the chaotic sequence have the ability to jump out of the local optimum.

\section{Statistical analysis}

Statistical analysis was performed using MedCalc version 13.0.0.0 (MedCalc software, Ostend, Belgium). The derived parameters are expressed as the mean \pm standard deviation. The Student's t-test was used to compare the estimated parameters between HCCs and background liver tissues. The box plot was used to assess the consistency of the estimated $k_{1}$ and $k_{3}$ for the different methods. The diagnostic performance of $k_{1}$ and $k_{3}$ among the three methods was compared using receiver operating characteristic (ROC) curve analysis. $P<0.05$ indicated significant differences. The fitting quality of the TACs among the three methods was compared using the Akaike information criterion (AIC) and the Bayesian 
information criterion (BIC), and a smaller value represents better curve fitting.

\section{Results}

\section{Kinetic parameters}

The kinetic parameters $\left(k_{1}, k_{2}, k_{3}\right.$ and $\left.k_{4}\right)$ and the HPI obtained by the three methods are shown in Table 1.

NLLS yielded a significant difference in HCCs due to its higher $k_{1}, k_{4}$ and the HPI than those in background liver tissue $(P=0.019, P<0.001$, and $P<0.001$, respectively), and $k_{2}$ and $k_{3}$ did not show a significant difference between HCCs and background liver tissue $(P=0.067$ and $P=0.411$, respectively).

For the GSA, $k_{1}, k_{3}, k_{4}$ and the HPI showed significant differences in distinguishing between HCCs and background liver tissue $(P=0.008, P<0.001, P<0.001$, and $P=0.019$, respectively), while $k_{2}$ did not reach significance. $(P=0.688)$.

For the DCGSA, $k_{1}, k_{3}$ and the HPI were significantly higher in HCCs than in background liver tissue $(P<0.001$, $P<0.001$, and $P<0.001$, respectively), and $k_{2}$ and $k_{4}$ were significantly lower in HCCs than in background liver tissue $(P<0.001$ and $P<0.001$, respectively).

The box plots of $k_{1}$ and $k_{3}$ estimated by the three methods are shown in Fig. 4. Compared with the GSA and NLLS, the DCGSA had a more compact data distribution and lower standard deviation of $k_{1}$ and $k_{3}$ in HCCs and in background liver tissues.

\section{Comparison of $k_{1}$ and $k_{3}$}

Figure 5 shows the ROC curves of $k_{1}$ and $k_{3}$ estimated by the three methods. For $k_{1}$, the diagnostic performance for differentiating HCCs from background liver tissue among the three methods was not significantly different (all $P>0.05$ ). For $k_{3}$, the DCGSA had higher diagnostic performance than the GSA and NLLS $(P=0.0024$ and $P=0.0001$, respectively); the diagnostic performance was not significantly different between the GSA and NLLS $(P=0.4420)$.

\section{TAC fit quality}

Figure 6 shows the mean and standard deviation of the $\mathrm{AIC}$ and BIC values for HCCs and background liver tissue using the DCGSA, GSA and NLLS. The DCGSA had the lowest AIC and BIC values among the three methods for HCCs, and the AIC and BIC values of the DCGSA were higher than those of the GSA and lower than those of NLLS for the background liver tissue.

\section{Discussion}

Conventional dynamic scans take $60 \mathrm{~min}$ or more, which is time-consuming, significantly limits the daily throughput of PET/CT scanners and the patients cannot remain immobile for a long period of time, thus it is not suitable for clinical settings. Based on previous study results, this paper used 5-min dynamic PET/CT for kinetic analysis to differentiate HCCs from background liver tissue [11, 12]. This study preliminarily introduced the GSA and improved kinetic parameter estimation with a dual-input dual-compartment model on dynamic PET/CT. The liver receives dual blood supplies from the hepatic artery and portal vein, which is neglected in traditional singleinput kinetic modeling, resulting in inaccuracy in kinetic parameter estimation [25, 26]. Moreover, hepatocytes contain glucose-6-phosphatase (G6P), which dephosphorylates ${ }^{18}$ FDG-6-phosphate $\left(k_{4}\right)$ [6]. In this paper, a reversible $\left(k_{4}>0\right)$ two-tissue three compartment model with dual blood input is used for kinetic modeling, and it can accurately describe the glucose metabolism of the liver.

Table 1 Parameter estimation results of the three methods

\begin{tabular}{|c|c|c|c|c|c|}
\hline & $k_{1}$ & $k_{2}$ & $k_{3}$ & $k_{4}$ & HPI (\%) \\
\hline \multicolumn{6}{|l|}{ NLLS } \\
\hline $\mathrm{HCCs}$ & $0.528 \pm 0.241$ & $0.535 \pm 0.200$ & $0.060 \pm 0.054$ & $0.061 \pm 0.014$ & $48.8 \pm 32.8$ \\
\hline Liver tissue & $0.362 \pm 0.197$ & $0.657 \pm 0.195$ & $0.074 \pm 0.051$ & $0.018 \pm 0.025$ & $10.9 \pm 15.7$ \\
\hline$P$ & 0.019 & 0.066 & 0.411 & $<0.001$ & $<0.001$ \\
\hline \multicolumn{6}{|l|}{ GSA } \\
\hline $\mathrm{HCCS}$ & $0.679 \pm 0.206$ & $0.754 \pm 0.183$ & $0.189 \pm 0.035$ & $0.111 \pm 0.046$ & $56.5 \pm 13.8$ \\
\hline Liver tissue & $0.545 \pm 0.073$ & $0.772 \pm 0.045$ & $0.161 \pm 0.036$ & $0.042 \pm 0.034$ & $21.8 \pm 7.3$ \\
\hline$P$ & 0.008 & 0.675 & 0.019 & $<0.001$ & $<0.001$ \\
\hline \multicolumn{6}{|l|}{ DCGSA } \\
\hline $\mathrm{HCCs}$ & $0.651 \pm 0.013$ & $0.592 \pm 0.012$ & $0.137 \pm 0.024$ & $0.064 \pm 0.003$ & $66.7 \pm 18.3$ \\
\hline Liver tissue & $0.628 \pm 0.015$ & $0.620 \pm 0.013$ & $0.075 \pm 0.024$ & $0.090 \pm 0.009$ & $31.0 \pm 9.2$ \\
\hline$P$ & $<0.001$ & $<0.001$ & $<0.001$ & $<0.001$ & $<0.001$ \\
\hline
\end{tabular}




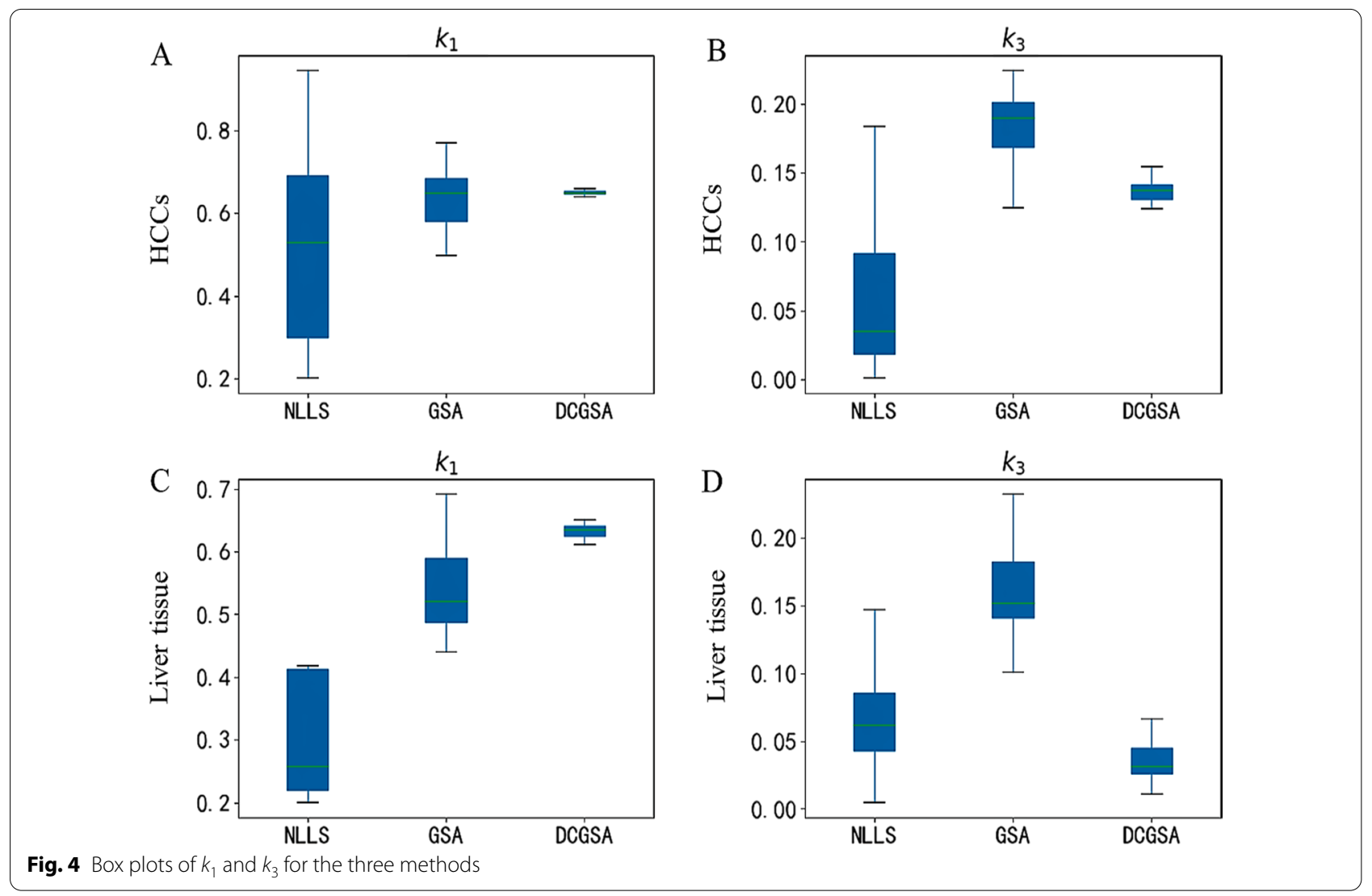

With the development of swarm intelligence optimization algorithms, some researchers have begun to use them to estimate kinetic models. Liu et al. [27] proposed an improved artificial immune network (AIN) for parameter estimation of a normal mouse liver kinetic model. Huang et al. [28] achieved kinetic analysis of PET images of Parkinson's disease by particle swarm optimization (PSO). Sara et al. [29] also demonstrated that the kinetic modeling of mouse kidneys can be estimated by ant colony optimization (ACO). The GSA is an optimization algorithm inspired by the theory of Newtonian gravity; its role in kinetic parameter estimation has attracted the attention of researchers. Ismail et al. [30] proposed a hybrid algorithm of PSO and the GSA for kinetic model parameter estimation of aspartate biochemical pathways.

This study preliminarily indicated that the GSA can estimate the parameters of the liver kinetic model and can distinguish HCCs from background liver tissue by $k_{1}$, $k_{3}, k_{4}$ and the HPI. To further enhance the global search capability and improve the strategy for jumping out of the local optimum to improve the accuracy of parameter estimation, an enhanced version, the DCGSA, is proposed in this paper, which showed significant differences in the kinetic parameters $k_{1}, k_{2}, k_{3}$, and $k_{4}$ and the HPI. NLLS is the most commonly used parameter estimation of kinetic models, but it can only distinguish HCCs from background liver tissue by $k_{1}, k_{4}$ and the HPI. Additionally, the statistical information criteria showed that the DCGSA achieved lower AIC and BIC values than those of the GSA and NLLS for HCCs and lower AIC and BIC values than those of NLLS for background liver tissue, which ensures improved TAC fitting quality.

Normal liver tissue is mainly supplied by the portal vein, which carries $70 \%-80 \%$ of the overall inflow, while $\mathrm{HCC}$ is a hypervascular tumor mainly supplied by the hepatic artery. The results of this study are consistent with those of previous studies, with the HPI of HCC being significantly higher than that of normal liver tissue $[10,12]$. The HPIs of HCC for NLLS, the GSA, and the DCGSA were $48.8 \pm 32.8,56.5 \pm 13.8$, and $66.7 \pm 18.3$, respectively, and the DCGSA was much closer to the expected clinical value. Most patients with HCC have a history of liver cirrhosis, which leads to an increase in the arterial blood supply of normal liver tissue [31]. When using the GSA and NLLS, the respective HPIs of normal liver tissue were $10.9 \pm 15.7$ and $21.8 \pm 7.3$ and were much lower than expected. However, the HPI of the DCGSA was $31.0 \pm 9.2$ and is closer to clinical practice.

Many glucose transporters (Gluts), which transport ${ }^{18} \mathrm{~F}$-FDG from the blood into hepatocytes, are distributed 

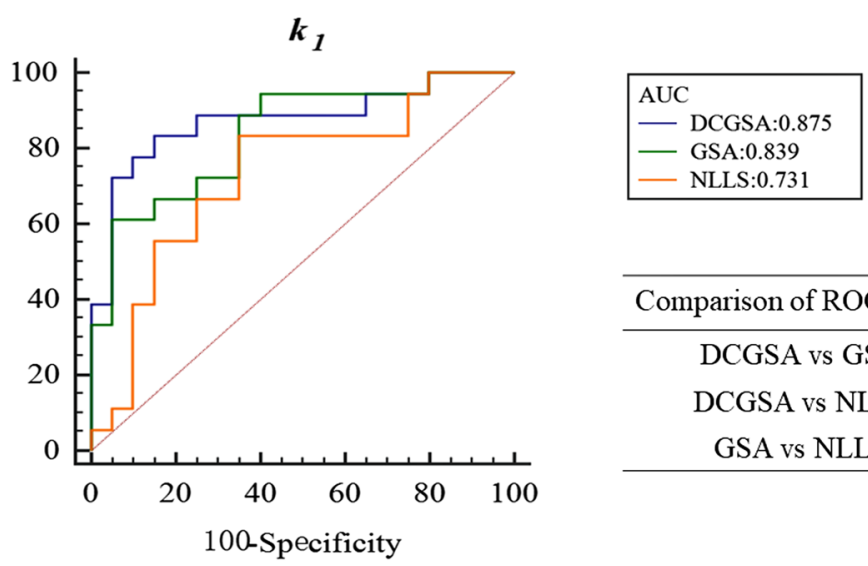

\begin{tabular}{cc}
\hline Comparison of ROC curves & $P$ \\
\hline DCGSA vs GSA & 0.667 \\
DCGSA vs NLLS & 0.207 \\
GSA vs NLLS & 0.209 \\
\hline
\end{tabular}
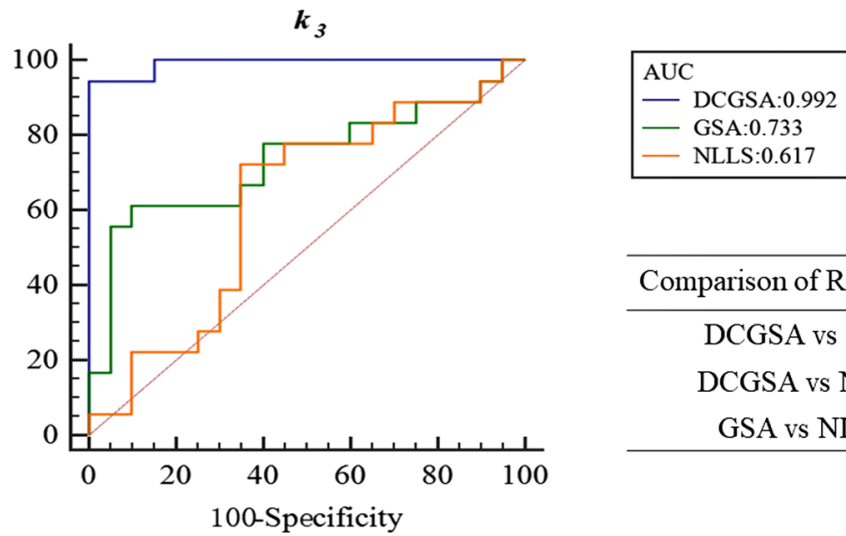

\begin{tabular}{cc}
\hline Comparison of ROC curves & $P$ \\
\hline DCGSA vs GSA & 0.002 \\
DCGSA vs NLLS & $<0.001$ \\
GSA vs NLLS & 0.442 \\
\hline
\end{tabular}

Fig. 5 Comparison of the ROC curves of $k_{1}$ and $k_{3}$
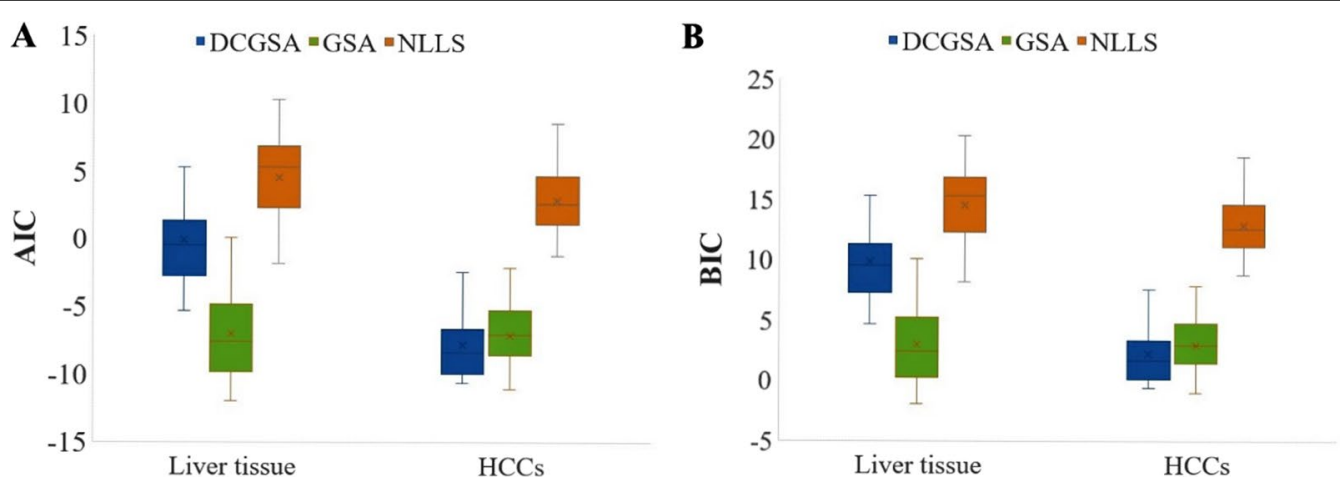

Fig. $6 \mathrm{AIC}$ and BIC values of TAC fitting by the three methods

on the plasma membrane of hepatocytes, and ${ }^{18} \mathrm{~F}$-FDG is phosphorylated to ${ }^{18} \mathrm{~F}$-FDG-6-phosphate by hexokinase (HK). The expression of Gluts is significantly higher in many cancer cells than in normal cells, and the uptake of glucose is increased. In addition, the expression of hexokinase and its affinity or functional activity of glucose phosphorylation is generally higher in cancer cells [32-35]. The transport rate $k_{1}$ and phosphorylation rate $k_{3}$, which are the parameters of major interest, are often used for quantitative analysis to achieve the diagnosis and assessment of HCC. Zuo et al. [36] reported the potential of using $k_{1}$ to noninvasively evaluate human liver inflammation. Geist et al. [13] compared four different kinetic models for kinetic modeling of $\mathrm{HCC}$ and 
demonstrated significant differences in $k_{3}$ between HCC and background liver tissue in all models.

In this study, $k_{1}$ and $k_{3}$ were greater in $\mathrm{HCC}$ than in background liver tissue by using the GSA and DCGSA, which is consistent with previous studies, implying increased Glut and HK activity in HCC [10, 37]. Meanwhile, the box plots showed higher consistency of $k_{1}$ and $k_{3}$ estimated with the DCGSA compared with NLLS and the GSA. Comparison of the ROC curves showed that the DCGSA had the highest diagnostic performance for $k_{3}$ and was significantly higher than that of the GSA and NLLS.

G6P activity and the dephosphorylation rate $k_{4}$ are higher in normal liver tissue than in HCC [6]. Our results showed that only $k_{4}$ of the DCGSA conformed to the above clinical study, but that of the GSA and NLLS did not.

In summary, ${ }^{18} \mathrm{~F}$-FDG kinetics can provide a comprehensive understanding of physiological systems and disease pathogenesis. The DCGSA can better estimate the kinetic parameters of the compartment model than the GSA and NLLS, which might play a significant role in clinical use for tumor characterization, monitoring the locoregional therapy and outcomes after resection.

This study has some limitations. First, the sample size of the experimental data is small. Second, there was a slight difference in ROI placement during TAC extraction, but we believe the influence was negligible. Third, this study did not explore lymph node involvement and other sites of metastasis, and we may explore other kinetic models in further studies. Fourth, the parameter estimation process is computationally expensive, and the proposed DCGSA might not be applicable to voxel level analysis for parametric images. It is necessary to improve the fitting algorithm to enhance the diagnostic performance and to develop more effective and efficient methods in our future research.

\section{Conclusions}

In this study, we demonstrated that the GSA can be used for parameter estimation of kinetic models on dynamic ${ }^{18} \mathrm{~F}-\mathrm{FDG} \mathrm{PET} / \mathrm{CT}$, and furthermore, the DCGSA was proposed to estimate the parameters more efficiently and reliably to distinguish HCC from background liver tissue.

\section{Abbreviations}

PET: Positron emission tomography; CT: Computed tomography; MRI: Magnetic resonance imaging; ${ }^{18} \mathrm{~F}-\mathrm{FDG}$ : Fluorine-18-fluorodeoxyglucose; HCC: Hepatocellular carcinoma; DCGSA: Dynamic chaotic gravitational search algorithm; GSA: Gravitational search algorithm; NLLS: Nonlinear least squares; HPI: Hepatic arterial perfusion index; SUV: Standardized uptake value; ROI: Regions of interest; TAC: Time-activity curve; OSEM: Ordered subsets expectation maximization; AIC: Akaike information criterion; BIC: Bayesian information criterion; AIN: Artificial immune network; PSO: Particle swarm optimization; ACO: Ant colony optimization; Gluts: Glucose transporters; HK: Hexokinase; G6P: Glucose-6-phosphatase.

\section{Acknowledgements}

Not applicable.

\section{Authors' contributions}

$J H$ and TW designed and performed the research and wrote the paper; $Y L$ and YD contributed to the analysis; SW critically revised the manuscript and supervised the report. All authors read and approved the final manuscript.

\section{Funding}

This work was sponsored in part by the High-level Talent Project of Health in Yunnan Province (No. D-2018011); the Ten Thousand People Plan in Yunnan Province (No. YNWR-QNBJ2018-243); the National Natural Science Foundation of China (Grant No. 81760306); and the Basic Research on Application of Joint Special Funding of Science and Technology Department of Yunnan ProvinceKunming Medical University (No. 2018FE001(-291)).

\section{Availability of data and materials}

The datasets generated and analyzed during the current study are not publicly available due to the security of data but are available from the corresponding author upon reasonable request.

\section{Declarations}

Ethics approval and consent to participate

The study was conducted in accordance with the Declaration of Helsinki, and the protocol was approved by the Ethics Committee of the First People's Hospital of Yunnan Province (No. 2017YYLH035). Prior informed consent to participate was obtained from all participants.

\section{Consent for publication}

Not applicable.

\section{Competing interests}

The authors declare that they have no competing interests.

\section{Author details}

${ }^{1}$ Faculty of Information Engineering and Automation, Kunming University of Science and Technology, Yunnan Key Laboratory of Artificial Intelligence, Kunming 650500, Yunnan, China. ${ }^{2}$ PET/CT Center, Affiliated Hospital of Kunming University of Science and Technology, First People's Hospital of Yunnan, Kunming 650031, China.

Received: 16 October 2021 Accepted: 24 January 2022

Published online: 06 February 2022

References

1. Sung H, Ferlay J, Siegel RL, Laversanne M, Soerjomataram I, Jemal A, Bray F. Global cancer statistics 2020: GLOBOCAN estimates of incidence and mortality worldwide for 36 cancers in 185 countries. CA Cancer J Clin. 2021;71(3):209-49.

2. Maluccio M, Covey A. Recent progress in understanding diagnosing and treating hepatocellular carcinoma. CA. 2012;62(6):394-9.

3. Shiomi S, Kawabe J. Clinical applications of positron emission tomography in hepatic tumors. Hepatol Res. 2011;41(7):611-7.

4. Rf A, Mc B, Sdv B. PET/CT in radiation oncology. Semin Oncol. 2019;46(3):202-9.

5. Tixier F, Vriens D, Cheze-Le Rest C, Hatt M, Disselhorst JA, Oyen WJ, de Geus-Oei LF, Visser EP, Visvikis D. Comparison of Tumor Uptake Heterogeneity Characterization Between Static and Parametric ${ }^{18}$ F-FDG PET Images in Non-Small Cell Lung Cancer. Journal of Nuclear Medicine, 2016,57(7).

6. Keiding S. Bringing physiology into PET of the liver. J Nucl Med. 2012;53(3):425-33. 
7. Ren-Cai Lu, She Bo, Gao W-T, Ji Y-H, Dong-Dong Xu, Wang Q-S, Wang S-B. Positron-emission tomography for hepatocellular carcinoma: current status and future prospects. World J Gastroenterol. 2019;25(32):4682-95.

8. Sarkar S, Corwin MT, Olson KA, Stewart SL, Liu CH, Badawi RD, Wang G. Pilot study to diagnose nonalcoholic steatohepatitis with dynamic ${ }^{18} \mathrm{~F}-$ FDG PET. Am J Roentgenol. 2019;212(3):529-37.

9. Wang J, Shao Y, Liu B, et al. Dynamic ${ }^{18}$ F-FDG PET imaging of liver lesions: evaluation of a two-tissue compartment model with dual blood input function. BMC Med Imaging. 2021;21(1):90.

10. Samimi R, Kamali-Asl A, Geramifar P, van den Hoff J, Rahmim A. Shortduration dynamic FDG PET imaging: optimization and clinical application. Physica Med. 2020;80:193-200.

11. Wang SB, Wu HB, Wang QS, Zhou WL, Tian Y, Li HS, Ji YH, Lv L. Combined early dynamic ${ }^{18} \mathrm{~F}$-FDG PET/CT and conventional whole-body ${ }^{18} \mathrm{~F}$-FDG PET/CT provide one-stop imaging for detecting hepatocellular carcinoma. Clin Res Hepatol Gastroenterol. 2015;39(3):324-30.

12. Wang S, Li B, Li P, Xie R, Wang Q, Shi H, He J. Feasibility of perfusion and early-uptake ${ }^{18} \mathrm{~F}$-FDG PET/CT in primary hepatocellular carcinoma: a dualinput dual-compartment uptake model. Japan J Radiol. 2021;6.

13. Geist BK, Wang J, Wang X, Lin J, Yang X, Zhang H, Li F, Zhao H, Hacker M, Huo L, Li X. Comparison of different kinetic models for dynamic ${ }^{18} \mathrm{~F}-\mathrm{FDG}$ PET/CT imaging of hepatocellular carcinoma with various, also dualblood input function. Phys Med Biol. 2020;65(4):045001.

14. Geist BK, Xing H, Wang J, Shi X, Zhao H, Hacker M, Sang X, Huo L, Li X. A methodological investigation of healthy tissue, hepatocellular carcinoma, and other lesions with dynamic ${ }^{68} \mathrm{Ga}-\mathrm{FAPI}-04 \mathrm{PET} / \mathrm{CT}$ imaging. EJNMMI Physics. 2021;8(1):8.

15. Mitra S, Mitra A. A genetic algorithms based technique for computing the nonlinear least squares estimates of the parameters of sum of exponentials model. Expert Syst Appl. 2012;39(7):6370-9.

16. Esmat R, Nezamabadi-pour H, Saryazdi S. GSA: a gravitational search algorithm. Inf Sci. 2009;179(13):2232-48.

17. Schmidt KC, Turkheimer FE. Kinetic modeling in positron emission tomography. J Nucl Med Allied Sci. 2002;46(1):70-85.

18. Alessandra B, Rizzo G, Veronese M. Deriving physiological information from PET images: from SUV to compartmental modelling. Clin Transl Imaging. 2014;2(3):239-51.

19. Z. Song, C. Tang, X. Chen, S. Song and J. Ji. A self-adaptive mechanism embedded gravitational search algorithm. In: 2019 12th International Symposium on Computational Intelligence and Design (ISCID). 2019, pp. 108-112

20. Mirjalili S, Lewis A. Adaptive gbest-guided gravitational search algorithm. Neural Comput Appl. 2014;25(7-8):1569-84.

21. Lei Z, Gao S, Gupta S, Cheng J, Yang G. An aggregative learning gravitational search algorithm with self-adaptive gravitational constants. Expert Syst Appl. 2020;152(2):113396.

22. Mirjalili S, Gandomi AH. Chaotic gravitational constants for the gravitational search algorithm. Appl Soft Comput. 2017;53:407-19.

23. Li W. An improved gravitational search algorithm for optimization problems. 2019 Chinese Control And Decision Conference (CCDC). 2019; vol. 4, pp. 1290-1293.

24. Gao S, Vairappan C, Wang Y, Cao Q, Tang Z. Gravitational search algorithm combined with chaos for unconstrained numerical optimization. Appl Math Comput. 2014;231:48-62.

25. Munk OL, Bass L, Roelsgaard K, Bender D, Hansen SB, Keiding S. Liver kinetics of glucose analogs measured in pigs by PET: importance of dualinput blood sampling. J Nuclear Med. 2001;42(5):795-801.

26. Wang G, Corwin MT, Olson KA, Badawi RD, Sarkar S. Dynamic PET of human liver inflammation: impact of kinetic modeling with optimizationderived dual-blood input function. Phys Med Biol. 2018;63(15):155004.

27. Liu L, Ding H, Huang HB. Improved simultaneous estimation of tracer kinetic models with artificial immune network based optimization method. Appl Radiat Isotope. 2016;107:71-6.

28. Huang C, Wang W, Tzen K, Lin W, Chou C. FDOPA kinetics analysis in PET images for Parkinson's disease diagnosis by use of particle swarm optimization. 2012 9th IEEE International Symposium on Biomedical Imaging (ISBI). 2012; pp. 586-589.

29. Garbarino S, Caviglia G, Brignone M, Massollo M, Sambuceti G, Piana M. Estimate of FDG excretion by means of compartmental analysis and ant colony optimization of nuclear medicine data. Comput Math Methods Med. 2013;2013:793142
30. Ismail AM, Mohamad MS, Abdul Majid H, Abas KH, Deris S, Zaki N, Mohd Hashim SZ, Ibrahim Z, Remli MA. An improved hybrid of particle swarm optimization and the gravitational search algorithm to produce a kinetic parameter estimation of aspartate biochemical pathways. Biosystems. 2017;162:81-9.

31. Koranda P, Myslivecek M, Erban J, Seidlová V, Husák V. Hepatic perfusion changes in patients with cirrhosis indices of hepatic arterial blood flow. Clin Nucl Med. 1999;24(7):507-10.

32. Haberkorn U, Ziegler SI, Oberdorfer F, Trojan H, Haag D, Peschke P, Berger MR, Altmann A, van Kaick G. FDG uptake, tumor proliferation and expression of glycolysis associated genes in animal tumor models. Nucl Med Biol. 1994;21(6):827-34.

33. Paudyal B, Paudyal P, Oriuchi N, Tsushima Y, Nakajima T, Endo K. Clinical implication of glucose transport and metabolism evaluated by ${ }^{18} \mathrm{~F}-\mathrm{FDG}$ PET in hepatocellular carcinoma. Int J Oncol. 2008;33(5):1047.

34. Paudyal B, Oriuchi N, Paudyal P, Higuchi T, Nakajima T, Endo K. Expression of glucose transporters and hexokinase II in cholangiocellular carcinoma compared using $\left[{ }^{18} \mathrm{~F}\right]-2$-fluro-2-deoxy-D-glucose positron emission tomography. Cancer Sci. 2008;99(2):260-6.

35. Amann T, Maegdefrau U, Hartmann A, Agaimy A, Marienhagen J, Weiss TS, Stoeltzing O, Warnecke C, Schölmerich J, Oefner PJ, Kreutz M, Bosserhoff AK, Hellerbrand C. GLUT1 expression is increased in hepatocellular carcinoma and promotes tumorigenesis. Am J Pathol. 2009;174(4):1544-52.

36. Zuo Y, Sarkar S, Corwin MT, Olson K, Badawi RD, Wang G. Structural and practical identifiability of dual-input kinetic modeling in dynamic PET of liver inflammation. Phys Med Biol. 2019;64(17):175023.

37. He YX, Guo QY. Clinical applications and advances of positron emission tomography with fluorine-18-fluorodeoxyglucose $\left({ }^{18} \mathrm{~F}-\mathrm{FDG}\right)$ in the diagnosis of liver neoplasms. Postgrad Med J. 2008;84(991):246-51.

\section{Publisher's Note}

Springer Nature remains neutral with regard to jurisdictional claims in published maps and institutional affiliations.

Ready to submit your research? Choose BMC and benefit from:

- fast, convenient online submission

- thorough peer review by experienced researchers in your field

- rapid publication on acceptance

- support for research data, including large and complex data types

- gold Open Access which fosters wider collaboration and increased citations

- maximum visibility for your research: over $100 \mathrm{M}$ website views per year

At BMC, research is always in progress.

Learn more biomedcentral.com/submissions 